\title{
Toxicity from treatment of neuroblastoma with ${ }^{131}$ I-meta-iodobenzylguanidine
}

\author{
James C. Sisson ${ }^{1}$, Raymond J. Hutchinson ${ }^{2}$, James E. Carey ${ }^{1}$, Brahm Shapiro ${ }^{1}$, Jon W. Johnson ${ }^{1}$, \\ Shirley A. Mallette ${ }^{1}$, and Donald M. Wieland ${ }^{1}$ \\ ${ }^{1}$ Division of Nuclear Medicine, Department of Internal Medicine and ${ }^{2}$ Division of Hematology-Oncology, \\ Department of Pediatrics, The University of Michigan Medical Center, Ann Arbor, MI 48109, 0028, USA
}

\begin{abstract}
Toxic effects from ${ }^{131} \mathrm{I}$-meta-iodobenzylguanidine $\left({ }^{131}\right.$ I-MIBG) treatments of neuroblastoma in six patients were recorded. The toxicity was largely confined to the hematologic system where circulating leukocytes and platelets regularly declined after each dose of ${ }^{131} \mathrm{I}$-MIBG; the values reached nadirs between three and seven weeks and recovered slowly over subsequent weeks. Prior bone marrow transplantation and infiltration of bone marrow by neuroblastoma appeared to make the hematologic system more vulnerable to the radiation. Dosimetry revealed greater absorbed radiation by the whole body than by the blood and bone marrow. These observations are explained by a relatively rapid exit of ${ }^{131}$ I-MIBG from the blood to other tissues (but not to the bone marrow). Since treatment of an aggressive and lethal tumor such as neuroblastoma should be pushed to a degree of toxicity, careful dosimetry in each case will be necessary as a guide to reach the point of maximally tolerable toxicity.
\end{abstract}

Key words: Meta-iodobenzylguanidine - Radiation toxicity - Neuroblastoma - Bone marrow

The radiopharmaceutical ${ }^{131}$ I-meta-iodobenzylguanidine ( $\left.{ }^{131} \mathrm{I}-\mathrm{MIBG}\right)$, concentrates in some cancers to a degree that therapeutic radiation is imparted. Reports describe beneficial effects from treatments using ${ }^{131} \mathrm{I}-\mathrm{MIBG}$ for malignant pheochromocytoma (Sisson et al. 1984; Hoefnagel et al. 1987), neuroblastoma and related tumors (Hoefnagel et al. 1987). Untoward responses to this form of therapy appear to be modest and well tolerated (Sisson et al. 1984; Hoefnagel et al. 1987).

Although tumors have diminished after administrations of ${ }^{131}$ I-MIBG, complete remissions have been uncommon and but a few months in duration; this has been particularly so in cases of neuroblastoma (Hoefnagel et al. 1987). It is possible that larger doses of ${ }^{131}$ I-MIBG will be more effective, but such an increase incurs the risk of toxic reactions.

Because neuroblastoma is a relatively common tumor in children, and because it is aggressive, and in stage IV almost always lethal, some untoward reactions from therapy are acceptable. Indeed, chemotherapy is ordinarily administered to patients with neuroblastoma to a point where

Offprint requests to. J.C. Sisson toxicity is evident; treatment with maximally tolerable doses is the rule. To obtain the best chance for success, a new form of treatment, such as ${ }^{131}$ I-MIBG, should probably be administered under the same rule.

We report here the toxic responses to therapeutic doses of ${ }^{131} \mathrm{I}-\mathrm{MIBG}$ in patients with neuroblastoma. The major toxicity has been in the hematologic system and appears to relate to absorbed radiation for the whole body rather than that for the blood.

\section{Materials and methods}

Patients. The patients were referred to the University of Michigan Medical Center for treatment of neuroblastoma by ${ }^{131} \mathrm{I}-\mathrm{MIBG}$. In each case the neuroblastoma was in stage IV and had previously been treated with one or more chemotherapeutic protocols. Eight patients had dosimetry measurements, however, 1 patient was excluded because an insufficient number of blood samples were obtained to enable calculation of absorbed radiation to her blood, and another patient was not included because his marrow function was already greatly diminished by the neuroblastoma and prior chemotherapy (platelets $30000 / \mathrm{mm}^{3}$ ) so that toxicity to the bone marrow from ${ }^{131} \mathrm{I}-\mathrm{MIBG}$ could not be judged. Thus, six patients with neuroblastoma formed the basis for this study. Patients 4 and 5 received 2 treatments, and patient 6 received 3 treatments with ${ }^{131} \mathrm{I}-\mathrm{MIBG}$, but complete data were available for only the 3rd treatment. All other patients received one treatment. Patient 1 developed infiltration of her bone marrow by neuroblastoma within weeks of treatment with ${ }^{131} \mathrm{I}-\mathrm{MIBG}$, and patients 2 and 5 had undergone bone marrow transplantation.

Methods. ${ }^{131}$ I-MIBG was synthesized for diagnostic studies and therapy by methods previously described (Sisson et al. 1984; Mangner et al. 1982). Diagnostic doses of ${ }^{131}$ I-MIBG were $1 \mathrm{mCi}(37 \mathrm{MBq})$ per $1.73 \mathrm{~m}^{2}$ of body surface area but did not exceed $1 \mathrm{mCi}$. Both diagnostic and therapeutic doses were infused intravenously over 90 min except in patient 5 whose diagnostic and therapeutic doses were infused over $24 \mathrm{~h}$.

Whole body dosimetry in children was calculated from measurements obtained at $2 \mathrm{~m}$ with a flat field scintillation probe containing a $2 \times 2 \mathrm{in}$. sodium iodide crystal and aimed at the xiphoid. For adult sized patients the distance from probe to patient was $2.5 \mathrm{~m}$. Readings were obtained from 
Table 1. Patient characteristics and absorbed radiation from ${ }^{131} \mathrm{I}-\mathrm{MIBG}$

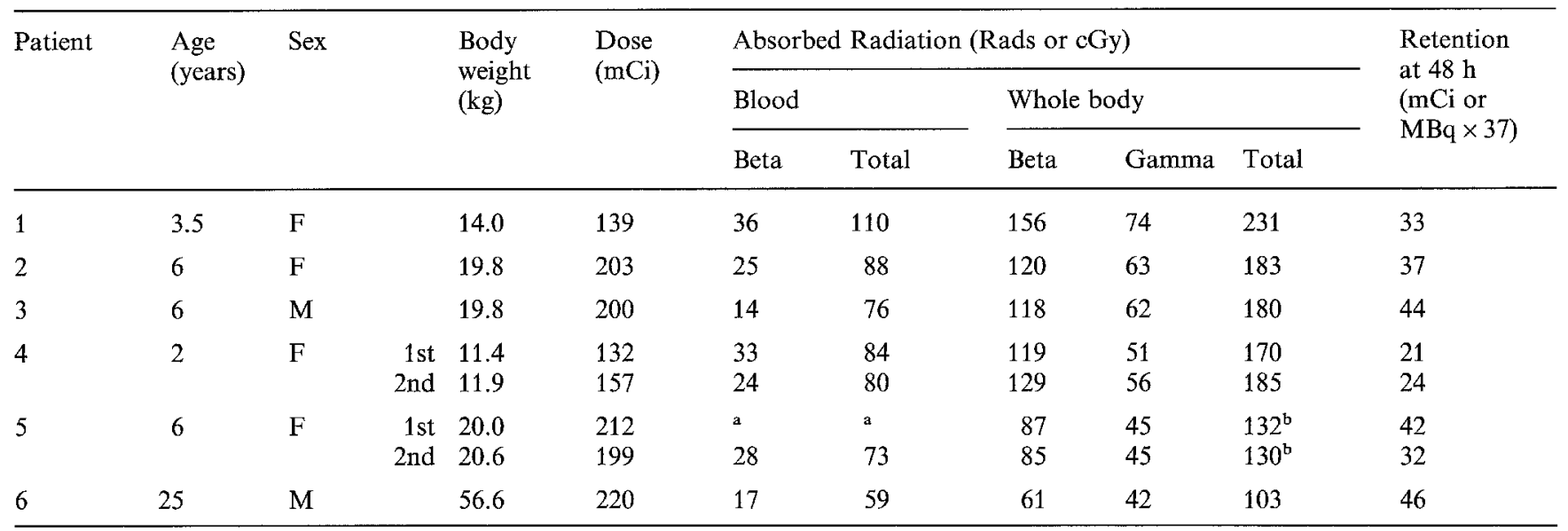

${ }^{a}$ Blood absorbed radiation not determined

${ }^{b}$ Whole body absorbed radiation measured from therapeutic dose because whole body counts not done and urine collection incomplete for diagnostic doses

both the anterior and posterior aspects of the patients to give a geometric mean. Body counts were obtained immediately following the infusion of the diagnostic dose of ${ }^{131} \mathrm{I}$ MIBG, $3 \mathrm{~h}$ after the infusion, and then daily for at least 4 days. An exception was patient 5 who had whole body radiation measured from her 2 therapeutic doses; body counts were not obtained from the diagnostic doses, and measurements based on urinary excretion rates of the diagnostic doses were vitiated by incomplete collections of urine. Calculation of whole body absorbed radiation dose was by a method previously described and modified (Leeper and Shimaoka 1980; Nostrand et al. 1986; Benua et al. 1962). In each patient, the whole body retention of tracer doses was compared with that obtained from a portable ionization chamber survey meter (Cutie pie) which gave readings of whole body radioactivity after the therapy dose (Thomas et al. 1980).

The absorbed dose of beta radiation in the whole body was calculated from the activity retained at the serial points of measurement assuming a uniform distribution of ${ }^{131} \mathbf{I}$ throughout the body (Berlin 1968). Errors arise because the ${ }^{131}$ I-MIBG is not uniformly distributed in patients, but these calculations give the best index available for comparision among patients. Total absorbed beta dose was obtained from the area below the individual data points. Tumor concentration of the radiopharmaceuticals was less than $2 \%$ of the dose, and therefore reciprocity of radiation from tumor to body was not considered.

Blood samples were obtained for counting radioactivity at the times of whole body counting. Absorbed beta radiation was calculated from the concentration of activity in blood over time, and the trapezoid areas determined for each time interval (Leeper and Shimaoka 1980; Nostrand et al. 1986; Benua et al. 1962). Absorbed gamma radiation by the blood was considered to be the same as that of the whole body. In three patients, bone marrow was aspirated for counting at the time the blood sample was obtained one day after the infusion of ${ }^{131}$ I-MIBG. The marrow aspirates contained no neuroblastoma cells on morphologic examination. Each marrow specimen was diluted by some blood, but, when the radioactivity per unit volume was compared with that in blood, a trend of more or less radioactivity could be ascertained.

Hematologic indices were obtained at one to two week intervals in the children, and at two to three month intervals in the adult patient.

\section{Results}

Excretion of ${ }^{131}$ I-MIBG was relatively rapid so that no patient retained more then $46 \mathrm{mCi}(1.7 \mathrm{GBq})$ by $48 \mathrm{~h}$ after dose administration (Table 1). Absorbed radiation by the whole body from ${ }^{131}$ I-MIBG was invariably greater than that by the blood (Table 1 ). Since gamma radiation to the blood was considered to be the same as that to the body, the differences between absorbed doses to the whole body and to the blood were most striking when the respective beta radiations were compared. When adsorbed radiation predicted from the diagnostic doses of ${ }^{131}$ I-MIBG was expressed as ratios of beta dose to blood divided by beta radiation to whole body, values were below 0.36 both at $24 \mathrm{~h}$ and for the total therapy (Table 2). Similar blood/body radiation ratios probably occurred when therapeutic doses were given since, following treatment with ${ }^{131} \mathrm{I}-\mathrm{MIBG}$, the total absorbed radiation to the body was similar to that predicted from the diagnostic dose (Table 2).

Although the aggregate body tissues received more radiation than blood from ${ }^{131} \mathrm{I}-\mathrm{MIBG}$, bone marrow was not a site of sequestration of this radiopharmaceutical. Marrow aspirates from three patients contained quantities of radioactivity similar to those in the respective blood specimens obtained at the same time (Table 2).

Since rates of excretion of ${ }^{131} \mathrm{I}-\mathrm{MIBG}$ were similar among the patients, the absorbed radiation was, for the most part, determined by the dose and body weight of the individual (and volume of distribution of the ${ }^{131} \mathrm{I}$ ). Thus, patient 6 , an adult weighing $56.6 \mathrm{~kg}$, received only $50 \%$ of the whole body radiation of patient 2 , a child weighing $19.8 \mathrm{~kg}$; yet, these 2 patients received similar doses of ${ }^{131} \mathrm{I}-$ MIBG, 220 and $203 \mathrm{mCi}(8.1$ and $7.5 \mathrm{GBq})$, respectively.

Except in patient 6, ${ }^{13}{ }^{1}$ I-MIBG therapies were associated with a substantial reduction in the circulating plate- 
Table 2. Relationships of absorbed radiation doses from ${ }^{131}$ I-MIBG

\begin{tabular}{lllll}
\hline Patient & $\begin{array}{l}\text { Blood/ } \\
\text { marrow }\end{array}$ & \multicolumn{2}{l}{$\begin{array}{l}\text { Beta dose: } \\
\text { blood/whole body }\end{array}$} & $\begin{array}{l}\text { Whole body } \\
\text { dosimetry } \\
\text { treatment dose/ } \\
\text { diagnostic dose }\end{array}$ \\
\cline { 3 - 5 } & $24 \mathrm{~h}$ & Total & 0.76 \\
\hline 1 & 1.08 & 0.35 & 0.23 & 0.93 \\
3 & 0.86 & 0.31 & 0.21 & 0.78 \\
4 & - & 0.14 & 0.12 & 0.80 \\
& 0.80 & 0.35 & 0.28 & 0.72 \\
5 & - & 0.23 & 0.19 & - \\
& - & - & - & - \\
6 & - & 0.28 & 0.32 & 0.93 \\
\hline
\end{tabular}

a Total whole body absorbed radiation calculated from diagnostic dose for treatment given: and directly from treatment dose by bedside dosimeter

lets (Table 3); the nadir, occurring at 6 weeks, was frequently less than $30 \%$ of the pretherapeutic values. Recovery occurred over many weeks (Fig. 1). This relative decline in platelets was generally greater than that of the leukocytes, although the circulating white cells were appreciably reduced in all neuroblastoma patients except patient 6 . At the nadir, the differential count was generally unchanged in the leukopenic specimens. However, patient 2 exhibited both a decline in total leukocytes and a selective neutropenia; her absolute neutrophil count at nadir was $384 / \mathrm{mm}^{3}$. Recovery from leukopenia also required weeks but was usually more rapid and complete than that for the thrombocytopenia (Fig. 1).

Patients were arranged in the tables according to absorbed dose for the whole body, but the changes in the circulating leukocytes and platelets did not follow this order. There was a relatively greater decline in both leukocytes and platelets in patients 1,2 and 5 than in the others (Table 3). Possibly the vulnerability of the hematologic sys- tem to radiation from ${ }^{131}$ I-MIBG in these patients related to the marrow transplantation in patients 1 and 5 and to the marrow infiltration by neuroblastoma that occurred shortly after treatment in patient 2 . The virtual absence of change in the leukocytes and platelets in patient 6 probably reflected the lower absorbed radiation dose in this adult man.

Comparison of the response in patient 4 , whose marrow was normal, with that in patient 5 , who had a marrow transplantation, is shown in Fig. 1. In neither patient was recovery of thrombocytopenia complete within 15 weeks after the initial treatment with ${ }^{131}$ I-MIBG. The nadir of leukocytes was reached slightly earlier than that of platelets.

No patient developed infection or bleeding in association with the decline in leukocytes and platelets. Patient 4 exhibited elevation in transminase enzyme values for some weeks after each therapy, probably reflecting some radiation hepatitis. The only other complication attributable to the therapy with ${ }^{131}$ I-MIBG was nausea and occasional vomiting for a day or two. Routine tests showed no abnormality in kidney function, and clinical appraisals found no disturbances in lung or heart. Concentrations of serum TSH, T4 cortisol and results of testing autonomic function (Sisson et al. 1984) showed no change.

\section{Discussion}

Treatments with substantial doses of a radiopharmaceutical such as ${ }^{131}$ I-MIBG, not unexpectedly, were associated with depression of circulating leukocytes and platelets, but the absorbed dose to the blood was less than that calculated for the whole body. Thus, based on the above data, toxicity to the bone marrow from ${ }^{131} \mathrm{I}-\mathrm{MIBG}$ will probably be more accurately predicted by measurements of radiation to the whole body than those of the blood. Indeed, if 200 rads to the blood were to be the limiting factor (Leeper and Shimaoka 1980), some of our patients would have received over $500 \mathrm{mCi}(18.5 \mathrm{GBq})$ in a single dose, a treatment that would probably have been lethal.

It is not clear how the bone marrow receives so much radiation. From the samples of marrow obtained, the

Table 3. Changes in circulating leukocytes and platelets following ${ }^{131} \mathrm{I}-\mathrm{MLBG}$

\begin{tabular}{|c|c|c|c|c|c|c|c|c|c|c|}
\hline Patient & $\begin{array}{l}\text { Whole body } \\
\text { total radiation } \\
\text { (RADS or cGy) }\end{array}$ & $\begin{array}{l}\text { Marrow } \\
\text { status }\end{array}$ & \multicolumn{4}{|c|}{ Leukocytes $\left(1000 / \mathrm{mm}^{3}\right)$} & \multicolumn{4}{|c|}{ Platelets $\left(1000 / \mathrm{mm}^{3}\right)$} \\
\hline 1 & 231 & Infiltration $^{\circ}$ & 6.7 & 2.6 & 0.39 & 6 & 235 & 70 & 0.30 & 5 \\
\hline 2 & 182 & Transplant & 5.1 & 1.6 & 0.31 & 7 & 201 & 52 & 0.26 & 5 \\
\hline 5 & $\begin{array}{l}132 \\
130\end{array}$ & Transplant & $\begin{array}{l}5.2 \\
4.7\end{array}$ & $\begin{array}{l}2.5 \\
1.6\end{array}$ & $\begin{array}{l}0.48 \\
0.34\end{array}$ & $\begin{array}{l}3 \\
2\end{array}$ & $\begin{array}{l}410 \\
267\end{array}$ & $\begin{array}{r}120 \\
63\end{array}$ & $\begin{array}{l}0.29 \\
0.24\end{array}$ & $\begin{array}{l}5-6 \\
6\end{array}$ \\
\hline 6 & 103 & Normal & 9.5 & 7.7 & 0.81 & $9^{\mathrm{d}}$ & 300 & 291 & 0.97 & 9 \\
\hline
\end{tabular}

\footnotetext{
a Nadir/before Rx values

b Interval between treatment and nadir

c Infiltration by neuroblastoma within weeks of therapy

${ }^{d}$ First post treatment sample at nine weeks
} 

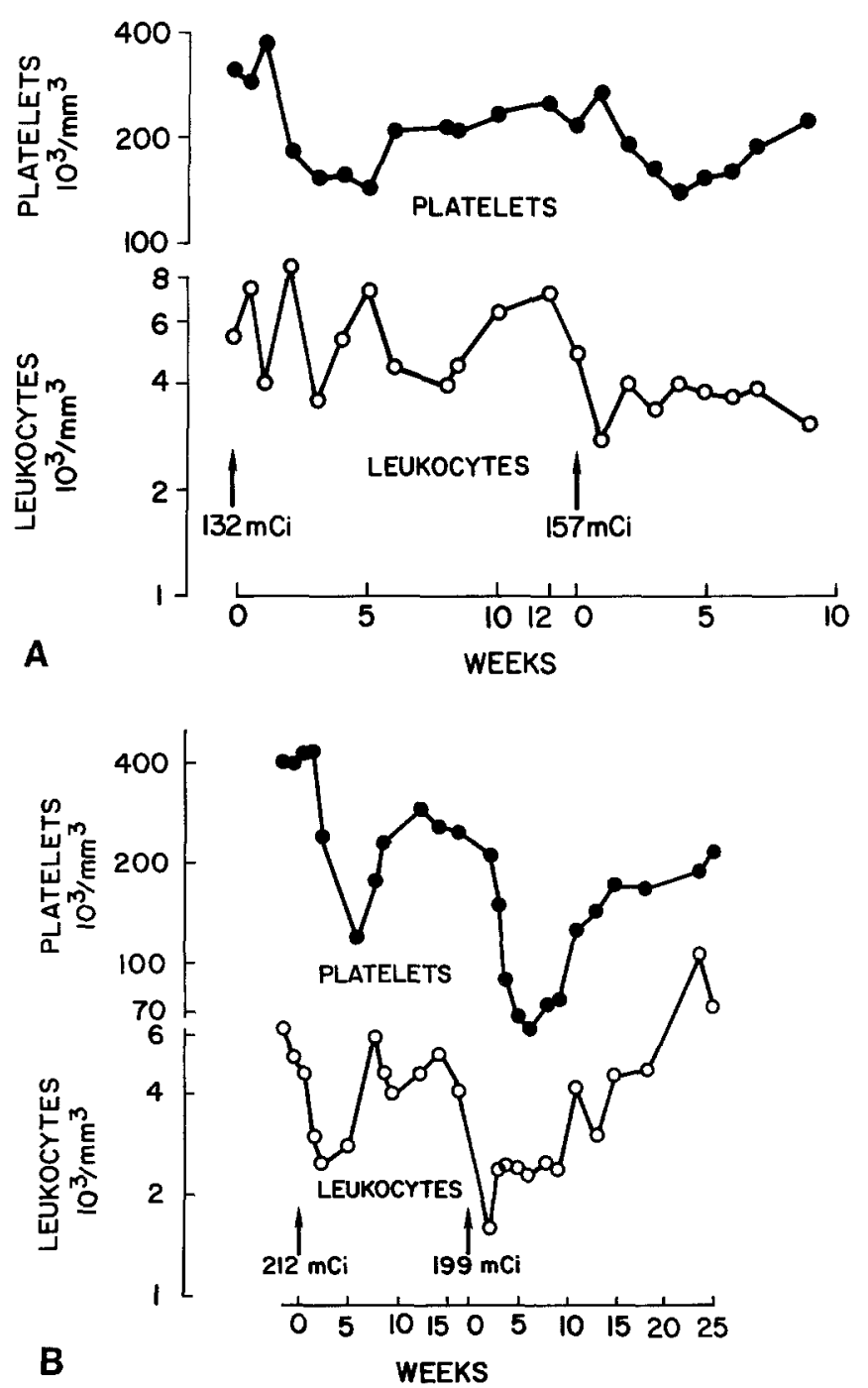

Fig. 1A, B. Circulating leukocyte and platelet values over time after treatments with ${ }^{131} \mathrm{I}-\mathrm{MIBG}$. A Patient 4 who had a normal bone marrow. The treatments with $132 \mathrm{mCi}(4.9 \mathrm{GBq})$ and $157 \mathrm{mCi}$ $(5.8 \mathrm{GBq}){ }^{13.1} \mathrm{I}-\mathrm{MIBG}$ imparted respectively, 170 and $185 \mathrm{rads}$ (cGy) to the body. B Patient 5 who had a bone marrow transplantation. The treatments with $212 \mathrm{mCi}(7.8 \mathrm{GBq})$ and $199 \mathrm{mCi}(7.4$ $\mathrm{GBq}){ }^{131} \mathrm{I}-\mathrm{MIBG}$ delivered, respectively, 132 and 130 rads (cGy) to the body, yet the declines in leukocytes and platelets were more marked than in $\mathbf{A}$

amount of radioactivity appears about the same or less than that in blood, at least at $24 \mathrm{~h}$ after the ${ }^{131} \mathrm{I}$-MIBG has been administered. Greater diffusion into the marrow at later times is possible but seems unlikely. Even at $24 \mathrm{~h}$ the absorbed dose to the body is greater than that to the blood indicating that exit of ${ }^{131}$ I-MIBG from blood to tissue is already taking place, but the tissues that sequester ${ }^{131}$ I-MIBG do not include the bone marrow. On the other hand, since ${ }^{131}$ I-MIBG is concentrated in platelets (Feldman et al. 1984), it is possible that certain marrow precursor cells, such as megakaryocytes, can selectively bind ${ }^{131} \mathrm{I}-$ MIBG to a level that would cause injury to these cells, but the quantity would not be apparent in an assay of overall bone marrow radioactivity.

The toxicity of ${ }^{131} \mathrm{I}$-MIBG was apparently made more prominent by bone marrow transplantation and by subsequent infiltration of marrow by neuroblastoma. All patients with neuroblastoma had received multiple courses of chemotherapy which may also have made the bone marrow more vulnerable to the effects of radiation.

Treatments of cancers by radiopharmaceuticals will probably follow the experience gained with chemotherapy. For aggressive and lethal neoplasms such as neuroblastoma, optimal effects will not be attained unless the treatment is pushed to toxic levels. Most often the limiting toxicity from radiopharmaceuticals will be depression of the bone marrow, and, as demonstrated in this report, measurements of absorbed doses of radiation will be required to determine the limiting guidelines for each new agent.

Acknowledgement. The authors are indebted to Mrs. Annise L. Johnson for expert typing of the manuscript. We also owe thanks to the many physicians who referred patients to us for treatment and to Dr. Sandford Jaques, Phillip Sherman and Dorothy Wu for counting blood and urine speciments for us.

The work was supported by the following Grants: AM 21477, DE-ACO2-76EVO2031, and 5 MO1RROO42 from the National Institutes of Health.

\section{References}

Benua RS, Cicale NR, Sonenberg M (1962) The relation of radioiodine dosimetry to results and complications in the treatment of metastic thyroid cancer. Am J Roentgenol Radiat Ther Nucl Med 87:171-182

Berlin I (1968) Polychthemia vera, leukemia and ${ }^{32} \mathrm{P}$ therapy. In: Wagner HN Jr (ed) Principles of Nuclear Medicine. W.B. Saunders, Philadelphia, pp 443-452

Feldman JM, Frankel N, Coleman RE (1984) Platelet uptake of the pheochromocytoma-scanning agent ${ }^{131}$ I-meta-iodobenzylguanidine. Metabolism 33:397-399

Hoefnagel CA, Voute PA, de Kraker J (1987) Radionuclide diagnosis and therapy of neural crest tumors using iodine-131 metaiodobenzylguanidine. J Nucl Med 28:308-314

Leeper RD, Shimaoka K (1980) Treatment of metastatic thyroid cancer. Clin Endocrinol Metab 9:383-404

Mangner TJ, Wu JL, Wieland DM (1982) Solid phase exchange radioiodination of aryl iodides. Facilitation by amonium sulfate. J Org Chem 47:1484-1488

Nostrand DV, Neutze J, Atkins F (1986) Side effects of "Rational Dose" iodine-131 therapy for metastatic well-differentiated thyroid carcinoma. J Nucl Med 1519-1527

Sisson JC, Shapiro B, Beierwaltes WH (1984) Radiopharmaceutical treatment of malignant pheochromocytoma. J Nucl Med 24: 197-206

Thomas SR, Maxon HR, Fritz KM (1980) A comparison of methods for assessing patient body burden following ${ }^{131} \mathrm{I}$ therapy for thyroid cancer. Radiology 137:839-842

Received August 11, 1987 / January 21, 1988 